\title{
A Novel Formulation Strategy to Deliver Combined DNA and VLP Based HPV Vaccine
}

\author{
Mohammad Uddin ${ }^{1}$, Brittany Henry ${ }^{1}$, Kewann D. Carter ${ }^{1}$, Monzurul A. Roni ${ }^{2}$, and Samir A. Kouzi ${ }^{3}$ \\ 1. College of Pharmacy, Larkin University, Miami, FL 33169. ${ }^{2}$ University of Illinois College of Medicine, Peoria, IL 61605. \\ 3. Levine College of Health Sciences, Wingate University, Wingate, NC 28174.
}

Received, October 11, 2019; Revised, October 13, 2019; Accepted, October 19, 2019; Published, October 20, 2019.

\begin{abstract}
Human papillomaviruses (HPV) are small, double-stranded DNA viruses that cause cervical cancer, the second most lethal cancer among women in the world. Currently, two vaccines are on the market for preventing HPV-caused cervical cancers and warts. Both are virus-like particle (VLP)-based vaccines. However, these vaccines have limitations; they are costly, have an invasive route of administration, require trained personnel to administer, need cold chain storage to preserve them, and most of all, they are preventive vaccines that do not have curative effects. Therefore, it is necessary to develop therapeutic HPV vaccines to facilitate the control of HPV-associated malignancies and to address all these issues. Recently there are DNA vaccines under investigation to prevent HPV. In general, DNA-based vaccines are better than or an excellent alternative to traditional vaccines since they can closely mimic live infections and can induce both antibody and cell-mediated immune responses. DNA vaccines involve the delivery of plasmid DNA (pDNA) which encodes the specific antigens. DNA vaccines have potential to be effective therapeutic tools against HPV infections. Combining the VLP-based and DNAbased vaccines can be highly effective as they can complement each other. VLP vaccines are more prone to mucosal immunity whereas DNA vaccines are more towards systemic immunity. In this article, we discuss an optimal formulation that will contain both type of vaccines, preventive and therapeutic. A film dosage form can be a good option which can be administered in buccal or sublingual routes for systemic action or in the vaginal area for local action to treat cervical cancer and to protect from future infection. Multiple vaccines in native form or in particulate form can be incorporated in film dosage forms. The film dosage form of vaccines can elicit both antibody-mediated (preventative) and cell-mediated (therapeutic) mechanisms. Film dosage forms are feasible to prepare for vaccine administration in the mouth cavity, GI tract, and vagina.
\end{abstract}

\section{INTRODUCTION}

Human papillomaviruses (HPV) are small, doublestranded DNA viruses that cause benign diseases such as warts or malignant diseases such as cancer. As of today, more than 150 different HPV types have been identified [1,2]. Out of all these types, some are considered as oncogenic or high-risk type such as HPV strains $16,18,31,33$ or 45 , which are responsible for the development of malignant cancers such as cervical cancer [3]. Cervical cancer is one of the most common cancers among women worldwide. HPV can also cause some other cancers such as tongue cancer and head-and-neck cancers $[4,5]$.

Currently, there are two vaccines on the market to prevent cervical cancer. These are Gardasil by Merck Pharmaceuticals, USA and Cervarix by GlaxoSmithKline Pharmaceuticals, UK. Both vaccines consist of the immunogenic L1 virus-like particles (VLPs) of HPV 16 and 18, with Gardasil containing additional 6 and 11 VLPs [6]. The vaccines are administrated intramuscularly and require multiple doses. These are expensive vaccines requiring cold storage, transfer facilities and trained personnel to administer. Apart from VLP-based vaccines, DNA-based vaccines have recently emerged as potentially promising approaches for vaccine development due to their safety profile, ease of preparation, and stability [6].

DNA seems highly effective against some malignant diseases. However, since DNA does not have the intrinsic ability to amplify or spread in transfected cells like viral vectors, DNA vaccines have limited immunogenicity.

Corresponding Author: Mohammad Uddin, PhD, Associate Professor, College of Pharmacy, Larkin University, Miami, FL 33169, USA. 
Therefore, it is important to develop innovative strategies to improve DNA vaccine potency. HPV therapeutic DNA vaccines will likely emerge as a significant approach that can be combined with existing forms of therapy, such as chemotherapy and radiation, leading to effective translation from bench to bedside for the control of HPV-associated malignancies [6]. Both the HIV DNA and VLP vaccines can induce antibodies as well as cytotoxic T cell responses $[7,8,9]$. However, DNA vaccines induce immune responses through direct contact with in vivo antigen synthesis whereas VLP vaccines directly present viral glycoproteins on the surface of an articulate antigen. As a result of their different mechanisms, immune responses induced by these two vaccine platforms are different. In a study by $\mathrm{Ye}$ et al, the immunogenicity of HIV Env-DNA and VLP vaccines were investigated for comparison and to evaluate whether a combination of these two vaccine platforms may complement each other when given as a mixture for inducing both antibody and CD8 T-cell responses [10]. This stirred the possibility of developing a common formulation that can contain both VLP-based and DNA-based cervical cancer vaccines where the two will complement each other and elicit a synergetic immunogenic effect.

Particulate form vaccines, especially the nanosized particulate vaccine, can address the limitations of VLP-based vaccines. Nano particulate VLP-based vaccines can be produced in an inexpensive and easier way and on a larger scale. These are stable at room temperature and therefore do not require cold chain storage. The handling or transfer of the vaccine is more cost effective and easier. In particulate form vaccines, no adjuvants are required, as the particle itself serves as an effective antigen delivery system and facilitates the uptake of antigen by antigen presenting cells. Particulate carriers can serve as an effective antigen delivery system that is able to enhance and/or facilitate the uptake of antigens by antigen-presenting cells (APCs) such as dendritic cells (DCs) or macrophages $[11,12]$. Particle-based antigen carriers can also serve as a depot for controlled release of the antigen, thereby increasing the availability of the antigen to the immune cells. It has been found that antigen release may enhance not only the level of the immune response but also its quality [ 13,14$]$. In addition, particle-based adjuvants possess the ability to modulate the type of induced immune responses when used alone or in combination with other immune-stimulatory compounds [15]. Particles can protect the integrity of antigens against degradation until delivered to the immune cells by selecting the appropriate polymer [16]. This is particularly important in oral vaccine formulations where antigens must be protected from the harsh acidic conditions of the stomach and enzymatic degradation in the gastrointestinal tract [17]. More importantly, particulate vaccines can potentially cross-present the antigen which is especially important to generate CD8+ T-cell responses against viral infections $[18,19]$. All these facts suggest that the particulate vaccine can be used globally especially in the resource-poor countries where the vaccines are more needed.

Recently, DNA vaccination has emerged as a promising alternative to traditional protein-based vaccines for the induction of protective immune responses. DNA vaccines offer several advantages over traditional vaccines, including increased stability, rapid and inexpensive production, and flexibility to produce vaccines for a wide variety of infectious diseases [20]. Also, DNA vaccines result in the intracellular production of the target antigen and subsequent presentation to the immune system. In turn, a more balanced T- and B-cell response is generated, which ultimately gives rise to populations of resident memory T-cells important in fighting mutating viral infections. DNA vaccines allow for rapid, large-scale production of antigen-specific vaccines and eliminate the need for cold chain storage and transportation, making them suited for rapidly emerging and pandemic diseases. However, the immunogenicity of DNA vaccines delivered as naked plasmid DNA is often weak due to degradation of the DNA by nucleases and inefficient delivery to immune cells. Therefore, biomaterialbased delivery systems based on micro- and nanoparticles that encapsulate plasmid DNA represent the most promising strategy for DNA vaccine delivery. In HPV infection, two oncogenic proteins, E6 and E7, are consistently co-expressed in HPV-expressing cervical cancers and are important in the induction and maintenance of cellular transformation [21]. Therefore, immunotherapytargeting E6 and/or E7 proteins may provide an opportunity to prevent and treat HPV-associated cervical malignancies. Chien et al has shown that a DNA vaccine can be used effectively against HPV infection [22]. 
Along with the antigen and the vaccine formulation, the route of administration and the delivery method also play a vital role in vaccine efficiency. In general, the oral route is the most patient compliant route and intramuscular is the most commonly used route for vaccine administration; however, the buccal area can be an attractive site for the delivery of vaccines because of its accessibility, avoidance of first pass effect, and immunological advantages over other mucosal routes of administration. A film dosage form for buccal administration dissolves with initial contact with saliva in the mouth cavity and facilitates the coating of the buccal surface, enhancing the delivery of the vaccine into the buccal mucosae. These films also assure dosing accuracy and elicit the induction of an effective immune response. Buccal mucosae are rich in immune cells such as dendritic cells and Langerhans cells which make them a suitable site for vaccine administration [23]. These cells can uptake the vaccines in original form or particulate form from the film designed for buccal delivery. Any type of vaccine can be formulated for buccal administration by optimizing the formulation in terms of types of antigen, size, surface charge or specific receptor ligands. Particulate formulation of the vaccine instead of pure form is most preferable to avoid the degradation of antigen by saliva. Several vaccines are under investigations to be administered in buccal mucosa using orally quickly dissolvable films [24].

\section{AVAILABLE VACCINES}

\section{Virus-like particles (VLP)-based vaccines}

Virus-like particles (VLPs) are capsids of viruses that does not contain DNA (Figure 1). VLPs are multiprotein structures that mimic the organization and conformation of authentic native viruses; but they lack the viral genome and can yield potentially safer and cheaper vaccine candidates for diseases caused by viruses [25]. Many infectious diseases, such as the common cold, influenza, rabies, measles, and different forms of diarrhea, hepatitis, yellow fever, polio, smallpox, and Acquired Immune Deficiency Syndrome (AIDS) are all caused by viruses [ 26]. A handful of prophylactic VLP-based vaccines are currently commercialized worldwide: Engerix (GSK) for hepatitis B virus and Cervarix (Merck) for human papillomavirus (HPV), and Recombivax HB(Merck) for hepatitis B virus and Gardasil (GSK) for human papillomavirus (HPV) are some examples. Other VLP-based vaccine candidates are in clinical trials or undergoing preclinical evaluation, such as, influenza virus, parvovirus, Norwalk and various chimeric VLPs [27]. There are many advantages to using VLPs in vaccines. They are excellent prophylactics because they are self-assembling bio-nanoparticles (20 to 60 $\mathrm{nm}$ in diameter) that expose multiple epitopes on their surface and faithfully mimic the native virions [28]. Unlike attenuated bacterial vaccines, the authentic and attenuated virions cannot be used as antigens in a prophylactic vaccine because they would contain oncogenic viral genomes that would be infectious [29]. VLPs eliminate this risk. Viruslike particles not only resemble authentic virions morphologically, but they also mimic virions immunologically which means they are able to induce high titers of neutralizing antibodies to conformational epitopes when vaccinated [30,31]. On the surface of VLPs there is an array of antigenic epitopes that mimic the surface of native virions more reliably than specific isolated subunits or subcomponents of the virus [32]. According to virology, VLPs are a class of subunit vaccines that differentiate themselves from soluble recombinant antigens by stronger protective immunogenicity associated with the VLP structure. Like parental viruses, VLPs can be either non-enveloped or enveloped, and they can form following expression of one or several viral structural proteins in a recombinant heterologous system [33]. The VLP can be produced in either a prokaryotic or eukaryotic expression system using target-encoding recombinant vectors, or in some cases can be assembled in cell-free conditions. Virus-like particles can be assembled by expressing the protein in a different medium such as mammalian cells, insect cells, yeast, or even bacteria [34]. Recent research also showed that it is possible to obtain plant derived VLPs in a cost-effective way [35].

VLPs represent an attractive concept for vaccine development [36, 37]. Like DNA vaccines, VLPs share the ability to be administered repeatedly to vaccinated individuals. The non-replicative nature of VLPs and their lack of viral genomic RNA make them safe for broad and repeated application. Earlier studies have shown that a viral glycoprotein presented in a highly repetitive form in virus particles is more potent in inducing a $\mathrm{B}$ cell response and antibody production than the same antigen presented in a poorly organized form [ 38,39$]$. In 
several studies, HIV VLPs have been shown to induce both neutralizing antibodies and CTL responses to HIV antigens [ 40,41].

\section{DNA Vaccine}

DNA-based vaccines are better than or an excellent alternative to traditional vaccines since they can almost mimic live infections and can induce both antibody- and cell-mediated immune responses. [42, $43,44]$. DNA vaccines involve the delivery of plasmid DNA (pDNA) which encodes the specifics antigen. This pDNA vaccine is driven by a eukaryotic promoter which results in the intracellular production of the target antigen [45]. Genetic/DNA immunization is a novel technique used to efficiently stimulate humoral and cellular immune responses to protein antigens. The direct injection of genetic material into a living host causes a small amount of its cells to produce the introduced gene products. This inappropriate gene expression within the host has important immunological consequences, resulting in the specific immune activation of the host against the gene-delivered antigen [46]. Since its early applications in the 1950's, DNA-based immunization has become a novel approach to vaccine development. Direct injection of naked plasmid DNA induces strong immune responses to the antigen encoded by the gene vaccine. Once the plasmid DNA construct is injected the host cells take up the foreign DNA, expressing the viral gene and producing the corresponding viral protein inside the cell. This form of antigen presentation and processing induced both major histocompatibility complex (MHC) class I and class II restricted cellular and humoral immune responses [47]. A plasmid vector that expresses the protein of interest (e.g. viral protein) under the control of an appropriate promoter is injected into the skin or muscle of the host. After uptake of the plasmid, the protein is produced endogenously and intracellularly processed into small antigenic peptides by the host proteases. The peptides then enter the lumen of the endoplasmic reticulum (E.R.) by membrane-associated transporters. In the E.R., peptides bind to MHC class I molecules. These peptides are presented on the cell surface in the context of the MHC class I. Subsequent CD8+ cytotoxic $\mathrm{T}$ cells (CTL) are stimulated and they evoke cell-mediated immunity. CTLs inhibit viruses through both cytolysis of infected cells and non-cytolysis mechanisms such as cytokine production [47]. The foreign protein can also be presented by the MHC class II pathway by APCs which elicit helper T-cells $(\mathrm{CD} 4+)$ responses. These CD4+ cells can recognize the peptides formed from exogenous proteins that were endocytosed or phagocytosed by APCs, then degraded to peptide fragments and loaded onto MHC class II molecules. Depending on the type of $\mathrm{CD} 4+$ cell that binds to the complex, B-cells are stimulated, and antibody production is stimulated. This is the same way traditional vaccines work [48].

DNA immunization offers many advantages over the traditional forms of vaccination. It can induce the expression of antigens that resemble native viral epitopes more closely than standard vaccines do since live attenuated and killed vaccines are often altered in their protein structure and antigenicity. Plasmid vectors can be constructed and produced quickly, and the coding sequence can be manipulated in many ways.

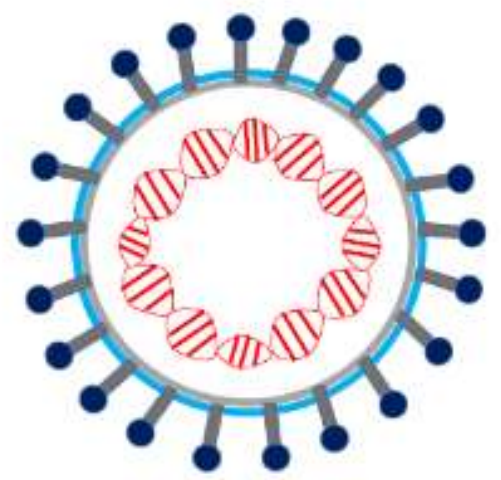

Human Papilloma Virus

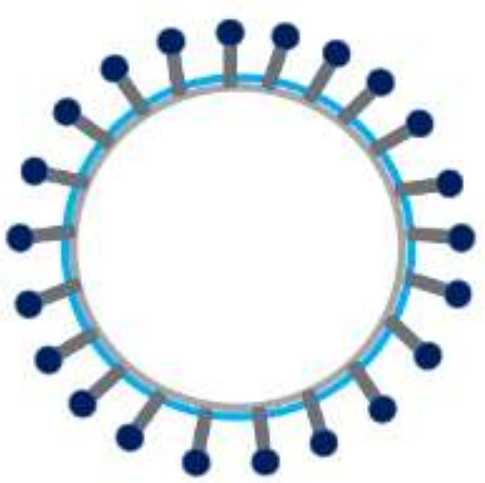

Virus-like particle

Figure 1. Human Papillomavirus and Virus-like particle (VLP). 
DNA vaccines encoding several antigens or proteins can be delivered to the host in a single dose, only requiring a microgram of plasmids to induce immune responses. Rapid and large-scale production are available at costs considerably lower than traditional vaccines, and they are also very temperature stable making storage and transport much easier. Another important advantage of genetic vaccines is their therapeutic potential for ongoing chronic viral infections. DNA vaccination may provide an important tool for stimulating an immune response in $\mathrm{HBV}, \mathrm{HCV}$ and HIV patients. The continued expression of the viral antigen caused by gene vaccination in an environment containing many APCs may promote successful therapeutic immune response which cannot be obtained by other traditional vaccines [47].

Furthermore, DNA vaccines eliminate the need for cold chain storage and transportation [49]. They can be quickly altered by manipulating the transgene sequence to adapt to new and fast-emerging diseases, [50] and are considered safer than traditional vaccines as the pathogen is not involved in vaccine synthesis. pDNA used in DNA vaccines can also be quickly and easily replicated and amplified in bacteria, allowing for accelerated production time frames, and the capability of large-scale production [51]. In addition, DNA vaccination is applicable to a range of viral, bacterial and parasitic diseases [42, $43,44]$. DNA vaccines are also uniquely suited for anticancer and antitumor therapies as their encoded antigen is produced intracellularly and introduced directly onto major histocompatibility complex (MHC) class I for antigen presentation to CD8bT cells, which are essential effector cells for cytolytic activity [52]. Although DNA can be used to raise immune responses against pathogenic proteins, certain microbes have outer capsids that are made up of polysaccharides. This limits the extent of the usage of DNA vaccines because they cannot substitute for polysaccharide-based subunit vaccines [53]. Several studies have shown that DNA vaccines can effectively induce both antibody and T-cell responses against their encoded antigens [54]. DNA immunization induces immune responses through both direct transfection of antigen presenting cells (APCs) and cross priming of APCs [ 55, 56] and offers several advantages over other vaccine platforms. First, the direct in vivo expression of antigens by DNA vaccination renders it more effective in eliciting cellular immune responses than protein-based vaccines, as in vivo synthesized antigens are processed and presented through both major histocompatibility complex I and II for inducing both CD4 and CD8 T-cell responses. Second, expression of the antigens over a long period of time after DNA vaccination may provide sustained stimulation of the immune system for inducing long-lasting immune responses [ 57]. Third, DNA vaccines can be applied repeatedly without inducing immune responses against the vector in contrast to recombinant viral vector-based vaccines. Currently scientists are working on developing DNA-based vaccines that will be highly effective and inexpensive. Liu et al has found that in vivo electroporation (EP) is a highly efficient nonviral method for enhancing DNA vaccine delivery and immunogenicity with hepatitis, when the site of immunization is the skin or muscle of animals and humans [58]. In another study, Bhowmik et al have found the role of albumin-based chitosan microparticles on enhancing immune response of plasmid DNA (pDNA) to a hepatitis-B surface antigen (HBsAg) vaccine after oral administration in mice. The results show an augmentation of both humoral and cellular immune responses for prolonged periods after immunization [59].

\section{Combination of DNA vaccines and VLP}

Developing a vaccine capable of inducing both protective humoral and cellular immune responses is a central challenge in vaccine research. The combination of DNA and VLP vaccines can address this challenge. Several studies have shown that DNA vaccines can effectively induce both antibody and Tcell responses against their encoded antigens [54, 55]. DNA immunization induces immune responses through both direct transfection of antigenpresenting cells (APCs) and cross priming of APCs $[55,56]$ and offers several advantages over other vaccine platforms. DNA vaccination may provide sustained stimulation of the immune system for inducing long-lasting immune responses [57]. DNA vaccines can be applied repeatedly without inducing immune responses against the vector in contrast to recombinant viral vector-based vaccines. On the other hand, Virus-like particles (VLPs) represent another attractive concept for vaccine development $[60,61,62]$. VLPs share with DNA vaccines the ability to be administered repeatedly to vaccinated individuals. The non-replicative nature of VLPs and their lack of viral genomic RNA make them safe for 
broad and repeated application. Since the assembly and arrangement of viral glycoproteins in VLPs resemble intact virions, they are likely to be more effective in inducing neutralizing antibodies as compared with soluble antigens. Earlier studies have shown that a viral glycoprotein presented in a highly repetitive form in virus particles is more potent in inducing $\mathrm{B}$ cell response and antibody production than the same antigen presented in a poorly organized form [ 63, 64]. In several studies, HIV VLPs have been shown to induce both neutralizing antibodies and CTL responses to HIV antigens [65, 66].

Combinations of DNA- and VLP-based vaccines have been extensively studied for HIV infections. It has been found that both humoral and cellular immune responses are critical for the control of HIV infections and replication. Yao et al have established a system to produce HIV and SIV viruslike particles containing high levels of viral Env proteins using the baculovirus expression system. The results of their study indicate that virus-like particles consisting of HIV structural proteins are an attractive vaccine platform for eliciting anti-viral immune responses, especially neutralizing antibody responses. They also have synthesized codonoptimized genes for HIV Env proteins and evaluated their immunogenicity. The results showed that combinations of VLP- and DNA-based vaccinations are promising for inducing strong cellular and neutralizing antibody responses against HIV [67].

While both the HIV DNA and VLP vaccines can induce antibodies as well as cytotoxic $\mathrm{T}$ cell responses, DNA vaccines induce immune responses through direct contact with in vivo antigen synthesis whereas VLP vaccines directly present viral glycoproteins on the surface of a particulate antigen $[68,69,70]$. As a result of their different properties, immune responses induced by these two vaccine platforms are likely to be different. In their study, Doan et al compared the immunogenicity of HIV Env-DNA and VLP vaccines to investigate whether a combination of these two vaccine platforms may complement each other when given as a mixture for inducing both antibody and CD8 T cell responses [70].

Gangadhara et al has shown the combination of DNA and VLP vaccines can enhance the immune response in HIV. They investigated humoral and cellular immune responses in mice after three sequential immunizations with DNA, a combination of DNA and virus-like particles (VLP), and VLP of recombinant modified vaccinia virus Ankara (rMVA) expressing HIV-1 89.6 gp120 envelope proteins (Env). The results showed an increase of over a 100-fold in Env-specific IgG antibody titers compared to three sequential immunizations with DNA and rMVA. This study suggests that a DNA and VLP combination vaccine with MVA is a promising strategy in enhancing the efficacy of DNA-rMVA vaccination against HIV-1. This study revealed that use of a combination of DNA and VLP vaccines as the first boost in the context of DNArMVA prime boost immunization could provide significantly enhanced humoral IgG antibody responses together with $\mathrm{T}$-cell immune responses [71].

Combination therapy of DNA and VLP vaccines is more effective than individual therapy. The mixture of DNA vaccines with VLP vaccines were more effective in responding against antigens in comparison to individual treatment. Individual treatments were only effective in targeting one source of treatment. Adjunct therapy targeted the same antigen at a higher rate, producing a greater response. In a recent study, Ye L. et al identified and compared individual treatment of Env DNA and VLPs. The immune response developed by immunization of HIV Env DNA vaccines and VLPs had both strengths and weaknesses. The individual immunization of HIV Env DNA vaccine produced a strong CD8 T-cell response. On the other hand, the individual immunization of the VLP vaccine induced high levels of antibody responses [69]. Unfortunately, both showed significant weakness in other areas. For example, HIV Env DNA showed relatively weak antibody responses and VLPs had minimal effect against CD8 T-cells. However, when combined, both HIV Env DNA and VLPs, there were improvements in effective treatment. Combination therapy minimized the weaknesses of individual treatment. Immunization that contained both the HIV Env DNA and VLP vaccines prompted an enhanced CD8 T-cell and antibody response. Therefore, it can be concluded that the immunization of a combination of DNA and VLP vaccines can elicit higher levels of both antibodies and CD8 T-cell responses [72].

In another study, Ding et al identified the significance of combination therapy of DNA vaccines and VLPs. VLP vaccines and DNA plasmids have been individually tested against highly pathogenic avian influenza viruses, but the 
combination of both vaccines against this virus has not been reported. By comparing various neutralizing antibody responses including immune protection elicited with DNA-VLP, DNA-DNA and VLP-VLP prime-boost approaches, the advantages of using a heterogeneous vector over the homologous approach were identified. All three prime-boost strategies protected against death. DNA-VLP and DNA-DNA have higher protection against infection symptoms, and DNA with VLP yields greater protection from infection. During this investigation of the effectiveness of DNA vaccines and VLPs, it was identified that there were greater neutralizing antibody responses, in addition to a higher rate of immune protection with heterologous DNA-VLP. It was also identified that this combination vaccine would show a lower incidence in mortality compared to homologous treatment [73].

A recent study identified heterologous DNAVLP as a prime-boost vaccine strategy. This combination treatment was effective in protecting against challenges on a broader spectrum. To further correlate the benefits, it is also evident in this study that DNA with VLP vaccine elicited heterosubtypic antibodies. Therefore, it appears that homologous neutralizing antibodies only protected against homologous and intra-subtype viruses. Heterologous antibodies were more effective in neutralizing and binding antibodies, which is enough to protect against heterosubtypic virus. This vaccine regimen also produced broad binding. Combinations of DNA and VLP neutralized antibody and T-cell responses that conferred broad protection against diverse challenge viruses. It suggests that this bivalent prime-boost strategy may become a candidate for a possible universal vaccine [74].

The evaluation of HIV-1 virus-like particles with DNA immune response intra-nasally identifies the benefits of another route of administration. In a study by Buonaguro et al, an in vivo mouse model was evaluated by intra-nasal administration of homologous VLPs protocol and heterologous DNA with VLP prime boost immunization. The study identified specific mucosal adjuvants that can effectively protect against antigens from the human immunodeficiency virus. The proposed use of heterologous DNA with virus-like particles protocol had the ability to increase the Env-specific humoral and cellular immune responses. When compared to the homologous administration the anti-gag responses increased in heterologous protocols. [75].
Ideally, combination therapy of DNA and VLPs stimulates neutralizing antibodies. In addition, this combination creates a robust cell-mediated immune response against various antibodies. In a study by Young et al, DNA vaccines were constructed to express virally regulated human immunodeficiency virus-like particles (VLP) to elicit broad-spectrum immune responses to multiple HIV-1 antigens. [76].

\section{Film-based HPV vaccines including VLP and DNA}

Oral films dissolve more rapidly over other dosage forms. It is very important to address the poor stability of liquid dosage forms, especially the aqueous formulations. Unlike the thin films, there is a need for great care during accurate measurement of the amount and shaking the bottle every time before administration may contribute to less acceptance by the patients [77]. Oral dissolving film is extensively useful for pediatric, geriatric, and psychiatric patients since it is easy to administer and avoids the risk of choking or suffocation, thus ensuring patient safety [78]. Oral films can also be beneficial for bedridden and non-cooperative patients as they can be administered easily and are hard to spit out. The rate of disintegration is rapid, allowing the drug to release, followed by the oromucosal absorption. Many drugs that undergo degradation in the GI tract are being administered by employing this route [79]. The film dosage form of vaccines can elicit both antibody-mediated (preventative) and cell-mediated (activate T-cells to destroy viruses) mechanisms. Film dosage forms are feasible to prepare vaccines for administration in the mouth cavity, GI tract, and vagina [80]. Currently, not many film dosage forms of drugs are on the market. However, there are some vaccines in the research phase which are in the film dosage form. These are: measles vaccine, live bacterial vaccines typhoid fever, salmonella, diphtheria toxoid, tetanus toxoid, poliomyelitis, and rotavirus vaccine which is designed to melt quickly in a baby's mouth prompting it to swallow the vaccine $[81,82]$. The dissolved medication is coated with a material to protect it in the child's stomach, and it is designed to release the vaccine in the small intestine where it should trigger an immune response to prevent a rotavirus infection [83],

Quickly soluble film dosage forms can also be used for therapeutic vaccines for local administration. One unique example could be for 
therapeutic use of HPV vaccines. This film can be used in the cervix area to create a local effect.

The possibility of using different types of vaccine in the film dosage form as a delivery system needs to be evaluated. Live vaccines in the film dosage form will not be a better choice as size and permeability and regeneration of antigen may cause side effects. Live attenuated influenza virus (A/PR/8 strain, H1N1) has been tested to be administered via the sublingual route and has been found to be safe and effective for inducing protective immune responses in mucosal and systemic compartments [84]. In an in vivo mouse study, Song et al. has shown that administration of an influenza vaccine produced virus-specific $\mathrm{IgG}$ in the serum and secretory $\operatorname{Ig} \mathrm{A}(\mathrm{s} \operatorname{Ig} \mathrm{A})$ in the respiratory mucosa. A single sublingual dose of $\mathrm{A} / \mathrm{PR} / 8$ virus prevented lung pathology induced by influenza virus challenge and provided a broad-range cross-protection against different influenza virus subtypes. Thereby, the risk of potential passage of the vaccine virus to the olfactory bulb was avoided by using the sublingual route since no viral RNA was detected in the brains of sublingually vaccinated mice, in contrast to mice that received the same vaccine intranasally [85]. Therefore, it is possible that vaccine administration in a film dosage form via the sublingual route can also elicit the protective immune response in both mucosal and systemic compartments if appropriate adjuvants and other auxiliary materials are used along with the vaccines. One approach to widening the choice of vaccination without the use of liveattenuated microorganisms is the development of adjuvants and delivery systems. These have recently been reviewed under the categories of mineral salts, oil-in-water emulsions, microbial derivatives, natural products, endogenous immunostimulatory factors, inert vehicles and lipid particulates [86]. Some of these are more appropriate for mucosal delivery for directly targeting the mucosal immune system (MIS). For example, alum, which is used in many parenteral vaccines, does not induce mucosal immunity. Delivery systems can also be used in combination with adjuvants and are designed to increase delivery, targeting, protection against degradation and controlled release of antigen at a site. The use of polymeric carriers such as chitosan, poly(lactic-co-glycolic acid) (PLGA) and poly lactic acid (PLA) have shown success as adjuvants and have been used as delivery carriers for vaccines in the form of microparticles and nanoparticles [87].
Reported uses of PLGA include spray-dried nanoparticles as carriers for diphtheria CRM-197 antigen and recombinant hepatitis B surface antigen, spray dried microparticles containing recombinant antigen $85 \mathrm{~B}$ against $\mathrm{TB}$ and wet microspheres containing hepatitis B surface antigen $[88,89,90]$.

In another study, DNA polymeric films (DNAPFs) were evaluated as an effective drug delivery system (DDS). They evaluated the growth behavior of oral squamous cell carcinoma (OSCC) cells on DNA-PFs. They also assessed morphological, biochemical, and cytometric features of the cells. MTT and resazurin assays showed reduction in cell viability related to increased DNA concentration in the DNA-PFs. The flow cytometry studies exhibited low cytotoxicity of DNA-PFs. The flow cytometric cell cycle analysis also exhibited average cell cycle phase distributions within later time points. This indicated that OSCC cell growth is maintained in the presence of DNA-PFs. DNA film supported cell growth. In addition, by incorporating active or photoactive compounds, DNA film was able to induce tissue regeneration. This may be useful in treating many diseases [91].

\section{CONCLUSIONS}

Human papillomaviruses (HPV) are lethal viruses that cause several cancers including cervical cancer which is an important health concern in the United States and throughout the world. There are two vaccines, Gardasil and Cervarix, that are available for HPV-related cancers and warts. But these vaccines have some major limitations that includes high cost, requirement of personnel to administer the vaccine, and need of cold chain storage to preserve and transport. These obstacles limit their use, particularly in resource-poor countries. Also, these vaccines sometimes show adverse effects to the body due to the intramuscular injection. Therefore, there is a great need for developing a new alternative HPV vaccine which will be cost effective and can significantly contribute to global public health. A film dosage form of HPV vaccine which is stated in this article, can be a good alternative. Film formulations are easy to prepare, have rapid onsets of action, and are inexpensive. Thus, it can be a very convenient tool for primary prevention of HPVcaused cancer and a realistic method to reduce the prevalence of this ailment globally. Also, since film dosage forms can be administered in multiple routes, 
this the best formulation to treat patient populations. Additionally, film dosage forms can incorporate either native form or particulate form of the HPV vaccine. Nano- or micro-particulate formulation vaccines have been found as very effective formulations which are more cost effective, easy to prepare and scale up, can be delivered via any route of administration, and can be preserved at room temperature. Particulate forms of vaccines can be administered as film dosage forms via buccal route. Anther advantage of film dosage forms is that the film can incorporate a combination of DNA and VLP-based vaccines. The previous studies support the theory that vaccines containing DNA and VLP components offer the most effective form of prevention. The immunization of combinations of DNA and VLP vaccines can elicit higher levels of both antibodies and CD8 T-cell responses. As a result of this synergistic effect, the combination therapy will limit replication of the infection. Thus, a film dosage form of vaccines that contains the particulate form combined DNA and VLP-based HPV vaccines can address the major challenges of current vaccines.

\section{CONFLICT OF INTEREST}

The authors have no relevant affiliations or financial involvement with any organization or entity with a financial interest in or financial conflict with the subject matter or materials discussed in the manuscript. This includes employment, consultancies, honoraria, stock ownership or options, expert testimony, grants or patents received or pending, or royalties.

\section{REFERENCES}

1. de Villiers EM, Fauquet C, Broker TR, Bernard HU, zur Hausen $\mathrm{H}$. Classification of papillomaviruses. Virology 2004;324: 7-27.

2. Munger K, Baldwin A, Edwards KM. Mechanisms of human papillomavirus-induced oncogenesis. J Virol. 2004;78: 11451-60.

3. Woodman CB, Collins SI, Young LS. The natural history of cervical HPV infection: unresolved issues. Nat Rev Cancer. 2007;7: 11-22.

4. Gillison ML. Human papillomavirus-associated head and neck cancer is a distinct epidemiologic, clinical, and molecular entity. Semin Oncol. 2004;31: 744-54.

5. Syrjanen S. Human papillomavirus (HPV) in head and neck cancer. J Clin Virol. 2005; 32(Suppl 1): S59-66.
6. Archana M, Shaw-Wei Tsen D, Chien-Fu Hung, and Wu T-C. Therapeutic HPV DNA vaccines. Expert Rev Vaccines. 2009; 8(9): 1221-1235.

7. Nabel G. J. Challenges and opportunities for development of an AIDS vaccine. Nature. 2001; 410(6831):1002-1007.

8. Deml L, Speth C, Dierich M. P, Wolf H, and Wagner R. Recombinant HIV-1 Pr55gag virus-like particles: potent stimulators of innate and acquired immune responses. Molecular Immunology. 2005; 42(2): 259-277.

9. Doan L, Li M, Chen C, and Yao Q. Virus-like particles as HIV-1 vaccines. Reviews in Medical Virology. 2005;15(2): 75-88.

10. Ye L, Wen Z, Dong K, Pan L, Bu Z, Compans RW, Zhang H, Yang C. Immunization with a Mixture of HIV Env DNA and VLP Vaccines Augments Induction of $\mathrm{CD} 8 \mathrm{~T}$ Cell Responses. $\mathrm{J}$ Biomed Biotechnol. 2010;497219.

11. Reddy ST, Rehor A, Schmoekel HG, Hubbell JA, Swartz MA. In vivo targeting of dendritic cells in lymph nodes with poly (propylene sulfide) nanoparticles. J Controlled Release. 2006; 112(1):26-34.

12. Walter E, Dreher D, Kok M. Hydrophilic poly (D, Llactide-co-glycolide) microspheres for the delivery of DNA to human-derived macrophages and dendritic cells. J Controlled Release. 2001; 76 (1-2):149-168.

13. Rice-Fichte AC, Arenas-Gambia AM, KahlMcDonagh MM, Ficht TA. Polymeric particles in vaccine delivery. Reviews particle-based properties, such as surface chemistry and sizes that will affect antigen presentation and processing by APCs. Curr Opin Microbiol. 2009;13(1):106-112.

14. Thomasin C, Corradin G, Men Y, Merkle HP, Gander B. Tetanus toxoid and synthetic malaria antigen containing poly(lactide)/poly(lactide-coglycolide) microspheres: importance of polymer degradation and antigen release for immune response. $\mathrm{J}$ Controlled Release. 1996; 41(1-2):131-145.

15. Mallapragada SK, Narasimhan B. Immunomodulatory biomaterials. Int J Pharm. 2008; 364(2):265-271.

16. Slütter B, Soema PC, Ding Z, Verheul R, Hennink W, Jiskoot W. Conjugation of ovalbumin to trimethyl chitosan improves immunogenicity of the antigen. $\mathrm{J}$ Controlled Release. 2010; 143(2):207-214.

17. O'Hagan DT. Microparticles and polymers for the mucosal delivery of vaccines. Adv Drug Deliv Rev. 1998; 34(2-3):305-320.

18. Jain S, Yap WT, Irvine DJ. Synthesis of proteinloaded hydrogel particles in an aqueous twophase system for coincident antigen and $\mathrm{CpG}$ oligonucleotide delivery to antigen-presenting cells. Biomacromolecules. 2005; 6(5):2590-2600.

19. Shen Z, Reznikoff G, Dranoff G, Rock K. Cloned dendritic cells can present exogenous antigens on 
both MHC class I and class II molecules. J Immunol. 1997; 158(6):2723-2730.

20. Farris E, Brown D, Ramer-Tait A, Pannier A. Microand nanoparticulates for DNA vaccine delivery.

21. Uddin N, Kouzi S, Hussain M. Strategies for Developing Oral Vaccines for Human Papillomavirus (HPV) Induced Cancer using Nanoparticle mediated Delivery System. J Pharm Pharm Sci. 2015; 18(2): $220-234$.

22. Chien-Fu H, Archana M, Ronald D. DNA vaccines for cervical cancer: from bench to bedside. Exp Mol Med. 2007; 39(6): 679-689.

23. Gala R, Popescu C, Knipp G, McCain R, Ubale R, Addo R, Bhowmik T, Kulczar C, D'Souza M. Physicochemical and Preclinical Evaluation of a Novel Buccal Measles Vaccine. AAPS PharmSciTech. 2017;18(2):283-292.

24. Uddin M, Allon A, Roni MA, Kouzi S. Overview and Future Potential of Fast Dissolving Buccal Films as Drug Delivery System for Vaccines. J Pharm Pharm Sci. 2019;22: 388 - 406.

25. Roldão A, Mellado MC, Castilho LR, Carrondo MJ, Alves PM. Virus-like particles in vaccine development. Expert Rev Vaccines. 2010;9(10):1149.

26. Uddin M. Virus-Like Particle: The Next Generation Vaccine? J Develop Drugs 2014; 3:1 DOI: 10.4172/2329-6631.

27. Roldão A, Mellado M, Castilho L, Carrondo M, Alves P. Virus-like particles in vaccine development. Expert Rev Vaccines. 2010; Oct9 (10):1149-76.

28. Zhao Q, Li S, Yu H, Xia N, Modis Y. Virus-like particle-based human vaccines: quality assessment based on structural and functional properties. Trends Biotechnol. 2013;31: 654-663.

29. Schiller JT, Hidesheim A. Developing HPV viruslike particle vaccines to prevent cervical cancer: a progress report. J Clin Virol. 2000;19: 67-74.

30. Kirnbauer R, Booy F, Cheng N, Lowy DR, Schiller J. Papillomavirus L1 major capsid protein selfassembles into virus-like particles that are highly immunogenic. Proc Natl Acad Sci U S A. 1992; 89: 12180-12184.

31. Rose R, Reichman R, Bonnez W. Human papillomavirus (HPV) type 11 recombinant virus-like particles induce the formation of neutralizing antibodies. J Gen Virol. 1994;75(8): 2075-2079.

32. Zhao Q, Li S, Yu H, Xia N, Modis Y. Virus-like particle-based human vaccines: quality assessment based on structural and functional properties. Trends Biotechnol. 2013;31: 654-663.

33. Kushnir N, Streatfield SJ, Yusibov V. Virus-like particles as a highly efficient vaccine platform: diversity of targets and production systems and advances in clinical development. Vaccine. 2012;31: 58-83.
34. Masavuli M, Wijesundara D, Torresi J, Gowans E, Grubor-Bauk B. Preclinical development and production of virus-like particles ss vaccine candidates for hepatitis C. Front Microbiol. 2017; 8: 2413.

35. Chen O, Lai H. Plant-derived virus-like particles as vaccines. Hum Vaccin Immunother. 2013; 9(1): 2649.

36. Noad R, Roy P. Virus-like particles as immunogens. Trends in Microbiology. 2003;11(9): 438-444.

37. Yao Z, Vzorov B, Yang C, Compans R. Virus-like particle and DNA-based candidate AIDS vaccines. Vaccine. 2003; 21(7-8):638-643.

38. Bachmann M, Rohrer U, Kundig T, Burki K, Hengartner $\mathrm{H}$, Zinkernagel $\mathrm{R}$. The influence of antigen organization on $\mathrm{B}$ cell responsiveness. Science. 1993; 262(5138):1448-1451.

39. Bachmann M, Hengartner H, Zinkernagel R. T helper cell-independent neutralizing $B$ cell response against vesicular stomatitis virus: role of antigen patterns in B cell induction? European Journal of Immunology. 1995;25(12):3445-3451.

40. Deml L, Kratochwil G, Osterrieder N, Kn"uchel R, Wolf $\mathrm{H}$, Wagner $\mathrm{R}$. Increased incorporation of chimeric human immunodeficiency virus type 1 gp120 proteins into Pr55(gag) virus-like particles by an Epstein-Barr virus gp220/350- derived transmembrane domain. Virology. 1997;235(1):1025.

41. Notka F, Stahl-Hennig C, Dittmer U, Wolf H, Wagner R. Accelerated clearance of SHIV in rhesus monkeys by virus-like particle vaccines is dependent on induction of neutralizing antibodies. Vaccine. 1999;18(3-4):291-301.

42. Stachyra A, Gora-Sochacka A, Sirko A. DNAvaccines against influenza. Acta Biochim Pol. 2014;61: 515-522.

43. Ingolotti M, Kawalekar O, Shedlock DJ, Muthumani $\mathrm{K}$, Weiner DB. DNA vaccines for targeting bacterial infections. Expert Rev Vaccines. 2010; 9:747-63.

44. Carvalho JA, Rodgers J, Atouguia J, Prazeres DM, Monteiro GA. DNA vaccines: a rational design against parasitic diseases. Expert Rev Vaccines. 2010;9: 175-91.

45. Langer B, Renner M, Scherer J, Schu le S, Cichutek K, Sudowe S, Reske-Kunz A. Safety Assessment of Biolistic DNA Vaccination in DNA delivery. New York Humana Press. 2013; 940:371-88.

46. Koprowski, H, Weiner D. DNA Vaccination/ Genetic Vaccination. Spriner-Verlag, Heidelberg.1998;198.

47. Encke, J., Jasper zu Putlitz, and Jack R. Wands. 1999. DNA Vaccines. Intervirology. 999;42: 117-124.

48. Schirmbeck R, Reimann J. Revealing the Potential of DNA-based Vaccination: Lessons Learned from the Hepatitis B Virus Surface Antigen. Biol. Chem. 2001; 382:543-552. 
49. Liu M. DNAvaccines: an historical perspective and view to the future. Immunol Rev. 2011; 239: 62-84.

50. Deering R, Kommareddy S, Ulmer J, Brito L, Geall A. Nucleic acid vaccines: prospects for non-viral delivery of mRNA vaccines. Expert Opin Drug Deliv. 2014;11: 885-99.

51. Kutzler M, Weiner D. DNA vaccines: ready for prime time? Nat Rev Genet. 2008;9: 776-88.

52. Anderson R, Schneider J. Plasmid DNA and viral vector-based vaccines for the treatment of cancer. Vaccine. 2007;25(Suppl 2):24-34.

53. American Academy of Microbiology. The Scientific Future of DNA for Immunization. 1996; http://www.asmusa.org/acasrc/Colloquia/dnareprt.pd f.

54. Donnelly J, Ulmer J, Shiver J, Liu M. DNA vaccines. Annual Review of Immunology. 1997; 15: 617-648.

55. Gurunathan S, Klinman D, Seder R. DNA vaccines: immunology, application, and optimization. Annual Review of Immunology. 2000; 18: 927-974.

56. D. J. Shedlock and D. B. Weiner. DNA vaccination: antigen presentation and the induction of immunity. Journal of Leukocyte Biology. 2000; 68(6): 793-806.

57. Dupuis M, Denis-Mize K, Woo K. Distribution of DNA vaccines determines their immunogenicity after intramuscular injection in mice.Journal of Immunology. 2000;165(5): 2850-2858.

58. Liu K, Ascenzi M, Bellezza C, Bezuidenhout A, Cote P, Gonzalez-Aseguinolaza G, Hannaman D, Luxembourg A, Evans C, Tennant B, Menne S. Electroporation Enhances Immunogenicity of a DNA Vaccine Expressing Woodchuck Hepatitis Virus Surface Antigen in Woodchucks. J Virol. 201;85(10):4853-4862.

59. Bhowmik T, D'Souza B, Uddin M, D'Souza MJ. Oral delivery of microparticles containing plasmid DNA encoding hepatitis-B surface antigen. J Drug Target. 2012;20(4):364-371.

60. Noad R, Roy P. Virus-like particles as immunogens,"Trends in Microbiology. 2003;11(9):438-444.

61. Yang C, Ye L, Compans R. Protection against filovirus infection: virus-like particle vaccines. Expert Review of Vaccines. 2008; 7(3): 333-344.

62. Yao Q, Bu Z, Vzorov A, Yang C, Compans R. Viruslike particle and DNA-based candidate AIDS vaccines.Vaccine. 2003; 21(7): 638-643.

63. Bachmann M, Rohrer U, Kundig T, Burki K, Hengartner $\mathrm{H}$, Zinkernagel $\mathrm{R}$. The influence of antigen organization on $\mathrm{B}$ cell responsiveness. Science. 1993;262(5138):1448-1451.

64. Bachmann M, Hengartner H, Zinkernagel R. T helper cell-independent neutralizing $\mathrm{B}$ cell response against vesicular stomatitis virus: role of antigen patterns in B cell induction? European Journal of Immunology. 1995;25(12):3445-3451.
65. Deml L, Kratochwil G, Osterrieder N, Kn"uchel R, Wolf $\mathrm{H}$, Wagner $\mathrm{R}$. Increased incorporation of chimeric human immunodeficiency virus type 1 gp120 proteins into Pr55(gag) virus-like particles by an Epstein-Barr virus gp220/350-derived transmembrane domain. Virology. 1997;235(1):1025.

66. Notka F, Stahl-Hennig C, Dittmer U, Wolf H, Wagner R. Accelerated clearance of SHIV in rhesus monkeys by virus-like particle vaccines is dependent on induction of neutralizing antibodies. Vaccine. 1999;18(3-4):291-301.

67. Yao Q, Bu Z, Vzorov A, Yang C, Compans RW. Virus-like particle and DNA-based candidate AIDS vaccines. Vaccine. 2003;21(7-8):638-643.

68. Nabel G. Challenges and opportunities for development of an AIDS vaccine. Nature. 2001; 410 (6831):1002-1007.

69. Deml L, Speth C, Dierich M, Wolf H, Wagner R. Recombinant HIV-1 Pr55gag virus-like particles: potent stimulators of innate and acquired immune responses. Molecular Immunology. 2005;42(2):259277.

70. Doan L, Li M, Chen C, Yao Q. Virus-like particles as HIV-1 vaccines. Reviews in Medical Virology. 2005;15(2) 75-88.

71. Gangadhara S, Kwon Y, Jeeva S, Quan F, Wang B, Moss B, Compans R, Amara R, Jabbar M, Kang S. Vaccination with combination DNA and Virus-Like particles enhances humoral and cellular immune responses upon boost with recombinant modified vaccinia virus ankara expressing human immunodeficiency virus envelope proteins. Vaccines (Basel). 2017;5(4).

72. Ye L, Wen Z, Dong K, Pan L, Bu Z, Compans R, Zhang $\mathrm{H}$, Yang C. Immunization with a Mixture of HIV Env DNA and VLP Vaccines Augments Induction of $\mathrm{CD} 8 \mathrm{~T}$ Cell Responses. J Biomed Biotechnol. 2010;2010:497219. doi: 10.1155/2010/497219. Epub 2010 May 25.

73. Ding H, Tsai C, Gutiérrez R, Zhou F, Buchy P, Deubel V, Zhou P. Superior neutralizing antibody response and protection in mice vaccinated with heterologous DNA prime and virus like particle boost against HPAI H5N1 virus. PLoS One. 2011;6(1):e16563.doi: 10.1371/journal.pone.0016563.

74. Jiang $\mathrm{W}$, Wang $\mathrm{S}$, Chen $\mathrm{H}$, Ren $\mathrm{H}$, Huang $\mathrm{X}$, Wang G, Chen Z, Chen L, Chen Z, Zhou P. A Bivalent Heterologous DNA Virus-Like-Particle Prime-Boost Vaccine Elicits Broad Protection against both Group 1 and 2 Influenza A Viruses. $\mathrm{J}$ Virol. 2017;91(9):2016-2052.

75. Buonaguro L, Devito C, Tornesello ML, Schröder U, Wahren B, Hinkula J, Buonaguro FM. DNA-VLP prime-boost intra-nasal immunization induces cellular and humoral anti-HIV-1 systemic and 
mucosal immunity with cross-clade neutralizing activity. Vaccine. 2007;25(32):5968-5977.

76. Young K, Smith J, Ross T. Characterization of a DNA vaccine expressing a human immunodeficiency virus-like particle. Virology. 2004;327(2):262-272.

77. Borges A, Silva C, Coelho J. Oral films: current status and future perspectives: I-galenical development and quality attributes. J Control Release. 2015;206: 1-9.

78. Amin P, Gangurde A, Alai P. Oral film technology: challenges and future scope for pharmaceutical industry. Int J Pharm Pharm Res. 2015;3(3):184-203.

79. Haque S, Sheela A. Development of polymer-bound fast-dissolving metformin buccal film with disintegrants. Int J Nanomed. 2015;10(Suppl 1):199.

80. Kraan H, Vrieling H, Czerkinsky C, et al. Buccal and sublingual vaccine delivery. J Control Release. 2014;190: 580-592.

81. Song J, Nguyen H, Cuburu N. Sublingual vaccination with influenza virus protects mice against lethal viral infection. Proceedings of the National Academy of Sciences. 2008;105(5):1644-1649.

82. Ferro V. Novel Approaches to Vaccine Research: Kerala (India). Research Signpost. Adjuvants and vaccine delivery systems. 2011;199-222.

83. Thomas C, Rawat A, Hope-Weeks L, et al. Aerosolized PLA and PLGA nanoparticles enhance humoral, mucosal and cytokine responses to hepatitis B vaccine. Mol Pharm. 2011;8(2):405-415.

84. Lu D, Garcia-Contreras L, Muttil P, et al. Pulmonary immunization using antigen $85-\mathrm{B}$ polymeric microparticles to boost tuberculosis immunity. AAPS J. 2010;12(3):338-347.

85. Muttil P, Prego C, Garcia-Contreras L. Immunization of guinea pigs with novel hepatitis $\mathrm{B}$ antigen as nanoparticle aggregate powders administered by the pulmonary route. AAPS J. 2010;12(3):330-337.

86. Thomas C, Gupta V, Ahsan F. Particle size influences the immune response produced by hepatitis $\mathrm{B}$ vaccine formulated in inhalable particles. Pharm Res. 2010;27(5):905-919.

87. Ahmed T, Aljaeid B. Preparation, characterization, and potential application of chitosan, chitosan derivatives, and chitosan metal nanoparticles in pharmaceutical drug delivery. Drug Des Devel Ther. 2016;10: 483.

88. Dinda S, Pattnaik G. Nanobiotechnology-based drug delivery in brain targeting. Curr Pharm Biotechnol. 2013;14(15):1264-1274.

89. Sun B, Xia T. Nanomaterial-based vaccine adjuvants. J Mater Chem B. 2016;4(33):5496-5509.

90. $\mathrm{Hu}$ Y, Hoerle R, Ehrich M. Engineering the lipid layer of lipid-PLGA hybrid nanoparticles for enhanced in vitro cellular uptake and improved stability. Acta Biomater. 2015;28: 149-159.

91. Jayme C, de Paula L, Rezende N, Calori I, Franchi L, Tedesco A. DNA polymeric films as a support for cell growth as a new material for regenerative medicine: Compatibility and applicability. Exp Cell Res. 2017;360(2):404-412. 